\title{
Lower bound of decay rate for higher-order derivatives of solution to the compressible fluid models of Korteweg type
}

\author{
Jincheng Gao, Zeyu Lyu(D) and Zheng-an Yao
}

\begin{abstract}
This paper concerns the lower bound decay rate of global solution for compressible Navier-Stokes-Korteweg system in three-dimensional whole space under the $H^{4} \times H^{3}$ framework. At first, the lower bound of decay rate for the global solution converging to constant equilibrium state $(1,0)$ in $L^{2}$-norm is $(1+t)^{-\frac{3}{4}}$ if the initial data satisfy some low-frequency assumption additionally. Furthermore, we also show that the lower bound of the $k(k \in[1,3])$ th-order spatial derivatives of solution converging to zero in $L^{2}$-norm is $(1+t)^{-\frac{3+2 k}{4}}$. Finally, it is proved that the lower bound of decay rate for the time derivatives of density and velocity converging to zero in $L^{2}$-norm is $(1+t)^{-\frac{5}{4}}$.
\end{abstract}

Mathematics Subject Classification. 35Q35, 35B40.

Keywords. Compressible Navier-Stokes-Korteweg system, Lower bound decay.

\section{Introduction}

In this paper, we are concerned with the lower bounds of decay rate for the global solution to the compressible Navier-Stokes-Korteweg system in three-dimensional whole space:

$$
\left\{\begin{array}{l}
\rho_{t}+\operatorname{div}(\rho u)=0, \\
(\rho u)_{t}+\operatorname{div}(\rho u \otimes u)-\mu \Delta u-(\mu+\nu) \nabla \operatorname{div} u+\nabla P(\rho)=\kappa \rho \nabla \Delta \rho,
\end{array}\right.
$$

where $t \geq 0$ is time, $x \in \mathbb{R}^{3}$ is spatial coordinate and the unknown functions $\rho=\rho(x, t)$ and $u=$ $\left(u_{1}, u_{2}, u_{3}\right)(x, t)$ represent density and velocity, respectively. The pressure $P(\rho)$ is a smooth function in a neighborhood of 1 with $P^{\prime}(1)>0$. The constant viscosity coefficients $\mu$ and $\nu$ satisfy the following physical conditions: $\mu>0,2 \mu+3 \nu \geq 0$. The constant capillary coefficient $\kappa$ satisfies $\kappa>0$. To complete the system (1.1), the initial data are given by

$$
\left.(\rho, u)(x, t)\right|_{t=0}=\left(\rho_{0}(x), u_{0}(x)\right) .
$$

Furthermore, as the space variable tends to infinity, we assume

$$
\lim _{|x| \rightarrow \infty}\left(\rho_{0}-1, u_{0}\right)(x)=0 .
$$

Korteweg-type models, supposing that the energy of the fluid depends on standard variables and the gradient of the density, are based on an extended version of nonequilibrium thermodynamics. The NavierStokes-Korteweg model, describing the dynamics of a liquid-vapor mixture with diffuse interphase and being used to model the motion of compressible fluid with capillary effect of materiel, originals from the work of van der Waals [23] and Korteweg [17], and the modern form was derived by Dunn and Serrin [8]. It should be noted that the system (1.1) will reduce to the well-known compressible Navier-Stokes system if the capillary coefficient satisfies $\kappa=0$. 
There are many studies on the well-posedness of solutions to the compressible fluid models of Korteweg type. For the one-dimensional case, many researchers have studied extensively; refer to $[3,5]$ and the references therein. Charve and Haspot [3] obtained the global strong solution in the case of Saint-Venant viscosity coefficients. Chen et al. [5] obtained the global existence of classical solutions with large initial data away from vacuum for the isothermal compressible fluid of Korteweg type under the condition that the viscosity coefficient and capillarity coefficient are dependent on the density. For the multidimensional case, Hattori and $\mathrm{Li}$ [12] showed the local existence of smooth solution with large initial data for the isothermal compressible fluid models of Korteweg type in $\mathbb{R}^{2}$. Later, Hattori and Li [13] showed the global existence of smooth solution with small initial perturbation for the isothermal compressible fluid models of Korteweg type in high dimensions in some Sobolev space. Danchin and Desjardins in [7] showed the existence and uniqueness of suitably smooth solutions in critical Besov space. This result was improved by Haspot [11] by showing the global existence of weak solution, while the initial data belong to the energy space. Bresch et al. [2] obtained global weak solutions for the isothermal Korteweg model in a periodic or strip domain without smallness of assumption on the initial data when the viscosity $\mu$ and the capillarity coefficient $\kappa$ are dependent on the density. For more results about the well-posedness result, the readers can refer to $[1,4,6,14-16,18,20,21]$ and the references therein.

The study for the asymptotic behavior of solution to the compressible Navier-Stokes-Korteweg equations has attracted many scholars' attention. First of all, the researchers in [20,24,25] established the time decay rates for the global solution under the $H^{N}(N \geq 3), H^{2}$ and $H^{1}$ framework, respectively. More precisely, Wang and Tan [25] established the global existence of solution and built the time convergence rates for the case $k=0,1$,

$$
\left\|\nabla^{k}(\rho-1)(t)\right\|_{H^{N+1-k}}+\left\|\nabla^{k} u(t)\right\|_{H^{N-k}} \leq C(1+t)^{-\frac{3+2 k}{4}} .
$$

Later, Gao et al. [10] proved that the convergence rates (1.4) come true when $k \in[0, N]$ with $N \geq 3$, which will be showed in Theorem 1.1. In order to obtain fast time convergence rates for the higher-order spatial derivatives of solution, Tan and Zhang [22] considered the case of initial data belonging to some negative Sobolev space rather than general $L^{1}$ space. More precisely, if the initial data $\left(\rho_{0}-1, u_{0}\right) \in$ $\left(H^{N+1} \cap \dot{H}^{-s}\right) \times\left(H^{N} \cap \dot{H}^{-s}\right)\left(N \geq 3\right.$ and $\left.s \in\left[0, \frac{3}{2}\right)\right)$, they established time decay rates

$$
\left\|\nabla^{k}(\rho-1)(t)\right\|_{H^{N+1-k}}+\left\|\nabla^{k} u(t)\right\|_{H^{N-k}} \leq C(1+t)^{-\frac{s+k}{2}},
$$

where $k=0,1,2, \ldots, N-1$. On the other hand, Li [18], Wang and Wang [24] studied the time decay rates of smooth solution and strong solutions under the smallness assumption on the potential external force in some Sobolev space, respectively. It should be noted that the decay rate (1.4) is called "optimal" in the sense that this rate of solution for the nonlinear part coincide with the decay rate of linearized one. Thus, the aim of this paper is devoted to providing lower bounds of decay rate (coincide with upper rate) for the global solution itself and its derivatives. In other words, this implies that the decay rate (1.4) obtained in [25] is really optimal.

Notation In this paper, the symbol $\nabla^{k}$ with an integer $k \geq 0$ stands for the usual any spatial derivatives of order $k$. For example, we define $\nabla^{k} v=\left\{\partial_{x}^{\alpha} v_{i}|| \alpha \mid=k, i=1,2,3\right\}, v=\left(v_{1}, v_{2}, v_{3}\right)$. We also denote the Fourier transform $\mathcal{F}(f):=\hat{f}$. Denote by $\Lambda^{s}$ the pseudo-differential operator defined by $\Lambda^{s} f=$ $\mathcal{F}^{-1}\left(|\xi|^{s} \hat{f}(\xi)\right)$. Denote $L^{2}\left(\mathbb{R}^{3}\right)$ and $H^{s}\left(\mathbb{R}^{3}\right)$ as the usual Lebesgue space and Sobolev space. For the sake of simplicity, we write $\int f \mathrm{~d} x:=\int_{\mathbb{R}^{3}} f \mathrm{~d} x$ and $\|(A, B)\|_{X}:=\|A\|_{X}+\|B\|_{X}$.

First of all, we recall the main results obtained in $[10,25]$ in the following:

Theorem 1.1. [10,25] Assume that the initial data $\rho_{0}-1 \in H^{N+1}$ and $u_{0} \in H^{N}$ for any integer $N \geq 3$ and there exists a small constant $\delta>0$ such that

$$
\left\|\rho_{0}-1\right\|_{H^{N+1}}+\left\|u_{0}\right\|_{H^{N}} \leq \delta
$$


then the solution $(\rho, u)$ of (1.1)-(1.3) satisfies for all $t \geq 0$

$$
\begin{aligned}
& \|(\rho-1)(t)\|_{H^{N+1}}^{2}+\|u(t)\|_{H^{N}}^{2}+\int_{0}^{t}\left(\|\nabla \rho(s)\|_{H^{N+1}}^{2}+\|\nabla u(s)\|_{H^{N}}^{2}\right) \mathrm{d} s \\
& \quad \leq C\left(\left\|\rho_{0}-1\right\|_{H^{N+1}}^{2}+\left\|u_{0}\right\|_{H^{N}}^{2}\right) .
\end{aligned}
$$

Furthermore, if $\left\|\left(\rho_{0}-1, u_{0}\right)\right\|_{L^{1}}$ is finite additionally, then it holds on

$$
\left\|\nabla^{\alpha}(\rho-1)(t)\right\|_{H^{N+1-\alpha}}+\left\|\nabla^{\alpha} u(t)\right\|_{H^{N-\alpha}} \leq C(1+t)^{-\frac{3+2 \alpha}{4}},
$$

for all $\alpha \in[0, N]$. Here, $C$ is a positive constant independent of time.

Next, we establish the lower bound of time decay rates for the global solution of (1.1)-(1.3) only under the $H^{4} \times H^{3}$ framework for the sake of simplicity.

Theorem 1.2. Denote $\varrho_{0}:=\rho_{0}-1$ and $m_{0}:=\rho_{0} u_{0}$. Assume that the Fourier transform $\mathcal{F}\left(\varrho_{0}, m_{0}\right)=$ $\left(\hat{\varrho_{0}}, \hat{m}_{0}\right)$ satisfies

$$
\left|\hat{\varrho}_{0}\right| \geq c_{0}, \quad \hat{m}_{0}=0,0 \leq|\xi| \ll 1,
$$

with $c_{0}$ a positive constant. Then, the global solution $(\rho, u)$ obtained in Theorem 1.1 has the decay rates for all $t \geq t_{*}$

$$
\begin{aligned}
c_{1}(1+t)^{-\frac{3+2 k}{4}} \leq\left\|\nabla^{k}(\rho-1)(t)\right\|_{L^{2}} \leq C_{1}(1+t)^{-\frac{3+2 k}{4}}, & k=0,1,2,3 ; \\
c_{1}(1+t)^{-\frac{3+2 k}{4}} \leq\left\|\nabla^{k} u(t)\right\|_{L^{2}} \leq C_{1}(1+t)^{-\frac{3+2 k}{4}}, & k=0,1,2,3 .
\end{aligned}
$$

Here, $t_{*}$ is a positive large time, and $c_{1}$ and $C_{1}$ are two positive constants independent of time.

Remark 1.1. The lower bounds of decay rates (1.9), (1.10) for the derivatives of density and velocity to the compressible Navier-Stokes-Korteweg system are obtained for the first time.

Remark 1.2. Although we only establish the time decay rates under the $H^{4} \times H^{3}$ framework in Theorem 1.2 , the method used here can be applied to the $H^{N+1} \times H^{N}(N \geq 3)$-framework. Under the condition (1.8), the global classical solution $(\rho, u)$ of the system (1.1) has the decay rates for all $t \geq t_{*}$

$$
\begin{aligned}
& c_{1}(1+t)^{-\frac{3+2 k}{4}} \leq\left\|\nabla^{k}(\rho-1)(t)\right\|_{L^{2}} \leq C_{1}(1+t)^{-\frac{3+2 k}{4}}, \quad k \in[0, N] ; \\
& c_{1}(1+t)^{-\frac{3+2 k}{4}} \leq\left\|\nabla^{k} u(t)\right\|_{L^{2}} \leq C_{1}(1+t)^{-\frac{3+2 k}{4}}, \quad k \in[0, N] .
\end{aligned}
$$

Here, $t_{*}$ is a positive large time, and $c_{1}$ and $C_{1}$ are two positive constants independent of time.

Remark 1.3. It should be pointed out that under the $H^{3}$-framework, the decay rates (1.9) and (1.10) for the global solution to the compressible Navier-Stokes equations can only be obtained under the condition that $k=0,1$ (see [9]). However, these decay rates for the solution to the Navier-StokesKorteweg equations can be obtained when $k=0,1,2,3$. The difference here is the appearance of Korteweg term $\kappa \rho \nabla \Delta \rho$ that will obtain enough dissipation for the density.

Finally, we will establish the lower bounds of decay rates for the time derivatives of solution to the compressible Navier-Stokes-Korteweg system (1.1).

Theorem 1.3. Assume the condition (1.8) holds on, then the global classical solution $(\rho, u)$ obtained in Theorem 1.1 satisfies for all $t \geq t_{*}$

$$
c_{2}(1+t)^{-\frac{5}{4}} \leq\left\|\partial_{t} u(t)\right\|_{L^{2}} \leq C_{2}(1+t)^{-\frac{5}{4}} .
$$

Furthermore, if there exists a small constant $\delta_{1}$ such that $\left\|u_{0}\right\|_{L^{1}} \leq \delta_{1}$, it holds on for all $t \geq t_{*}$

$$
c_{2}(1+t)^{-\frac{5}{4}} \leq\left\|\partial_{t} \rho(t)\right\|_{L^{2}} \leq C_{2}(1+t)^{-\frac{5}{4}} .
$$

Here, $t_{*}$ is a positive large time, and $c_{2}$ and $C_{2}$ are two positive constants independent of time. 
Remark 1.4. The lower bounds of decay rates for the time derivatives of density and velocity for the compressible Navier-Stokes-Korteweg system in the $L^{2}$ norm are obtained for the first time.

Now we make comments on the analysis of this paper. At first, we give the lower bound of decay rate for the higher-order spatial derivative of solution to the compressible Navier-Stokes-Korteweg equations (1.1). Define $U$ and $U_{l}$ as the solution to the nonlinear and linearized problems, respectively. Define $U_{\delta}:=U-U_{l}$, then we have for any integer $k$

$$
\left\|\nabla^{k} U\right\|_{L^{2}} \geq\left\|\nabla^{k} U_{l}\right\|_{L^{2}}-\left\|\nabla^{k} U_{\delta}\right\|_{L^{2}}
$$

For $\alpha_{l, k}<\alpha_{\delta, k}$, if the solutions $U_{l}$ and $U_{\delta}$ satisfy

$$
\left\|\nabla^{k} U_{l}\right\|_{L^{2}} \geq C_{l, k}(1+t)^{-\alpha_{l, k}}, \quad\left\|\nabla^{k} U_{\delta}\right\|_{L^{2}} \leq C_{\delta, k}(1+t)^{-\alpha_{\delta, k}}
$$

where $C_{l, k}$ and $C_{\delta, k}$ are positive constants independent of time. For large time $t$, it holds on

$$
\left\|\nabla^{k} U\right\|_{L^{2}} \geq C_{l, k}(1+t)^{-\alpha_{l, k}}-C_{\delta, k}(1+t)^{-\alpha_{\delta, k}} \geq C(1+t)^{-\alpha_{l, k}},
$$

where $C$ is a positive constant that independent of time. It is easy to obtain the lower bound of decay rate for the linearized part by applying the spectral analysis to the semigroup for the linearized NavierStokes-Korteweg system (2.4); see Proposition 2.1 in Sect. 2. Therefore, it is significant to obtain upper bound of decay rate of $\nabla^{k} U_{\delta}$. To achieve this goal, we establish the energy estimates, see (2.21) and (2.22) in Lemma 2.2. Then, with the help of the upper bound decay estimate (1.7), the upper bound of decay rate for $U_{\delta}$ can be obtained. While taking the case $k \geq 1$ into account, we utilize the Fourier Splitting method developed by Schonbek [19] to obtain the upper bound of decay rate for $\nabla^{k} U_{\delta}$.

Next, the upper and lower bounds of decay rate for the time derivative of velocity can be obtained by using the equation and lower bound of first-order spatial derivative. If we use the transport equation to obtain the lower bound of decay rate for the time derivative of density, we need to get the lower bound for the quantity divergence of velocity(i.e., divu). To achieve this target, we need to assume the smallness for the initial velocity in $L^{1}$.

The rest of this paper is organized as follows. In Sect. 2, we establish the lower bound of decay rate for the solution itself and derivative, and then, we establish the upper and lower bounds of decay rate for the time derivatives of solution. In Sect. 3, we prove technical estimates used in Sect. 2.

\section{Lower bounds of decay for spatial derivative}

In this section, we will address the lower bound of decay rates for the solution itself and its derivative. To this end, the upper decay rates for the difference between the nonlinear and linearized parts will be established. Finally, we address the upper decay rate of solution for the higher-order spatial derivative.

\subsection{Lower bounds of decay for spatial derivative}

In this subsection, we will establish optimal time decay rates of solution for the compressible NavierStokes-Korteweg equations (1.1)-(1.3). Let us denote $\varrho:=\rho-1, m:=\rho u$. For simplicity, we take $P^{\prime}(1)=$ 1 ; then, we rewrite (1.1) in the perturbation form as follows:

$$
\left\{\begin{array}{l}
\varrho_{t}+\operatorname{div} m=0 \\
m_{t}-\mu \Delta m-(\mu+\nu) \nabla \operatorname{div} m+\nabla \varrho-\kappa \nabla \Delta \varrho=-\operatorname{div} S,
\end{array}\right.
$$

where the function $S=S(\varrho, u)$ is defined as

$$
\begin{aligned}
S= & (\varrho+1) u \otimes u+\mu \nabla(\varrho u)+(\mu+\nu) \operatorname{div}(\varrho u) \mathbb{I}_{3 \times 3} \\
& +(P(1+\varrho)-P(1)-\varrho) \mathbb{I}_{3 \times 3}-\kappa \varrho \nabla^{2} \varrho+\frac{1}{2} \kappa|\nabla \varrho|^{2} I_{3 \times 3} .
\end{aligned}
$$


The initial data are given as

$$
\left.(\varrho, m)(x, t)\right|_{t=0}=\left(\varrho_{0}, m_{0}\right)(x) \rightarrow(0,0) \quad \text { as } \quad|x| \rightarrow \infty .
$$

In order to obtain the lower decay estimate, we need to analysis the linearized part:

$$
\left\{\begin{array}{l}
\partial_{t} \varrho_{l}+\operatorname{div} m_{l}=0, \\
\partial_{t} m_{l}-\mu \Delta m_{l}-(\mu+\nu) \nabla \operatorname{div} m_{l}+\nabla \varrho_{l}-\kappa \nabla \Delta \varrho_{l}=0,
\end{array}\right.
$$

with the initial data

$$
\left.\left(\varrho_{l}, m_{l}\right)(x, t)\right|_{t=0}=\left(\varrho_{0}, m_{0}\right)(x) \rightarrow(0,0) \quad \text { as } \quad|x| \rightarrow \infty .
$$

Here, we assume initial data for the linearized system (2.4) is the same as the original problem (2.1).

Proposition 2.1. Let $\varrho_{0} \in H^{4}\left(\mathbb{R}^{3}\right) \bigcap L^{1}\left(\mathbb{R}^{3}\right), m_{0} \in H^{3}\left(\mathbb{R}^{3}\right) \cap L^{1}\left(\mathbb{R}^{3}\right)$. Assume that the Fourier transform $\mathcal{F}\left(\varrho_{0}, m_{0}\right)=\left(\varrho_{0}, \hat{m}_{0}\right)$ satisfies $\left|\hat{\varrho}_{0}\right| \geq c_{0},\left|\hat{m}_{0}\right|=0,0 \leq|\xi| \ll 1$ with $c_{0}$ a positive constant, then there exists a positive large time $t_{*}$ such that for $t \geq t_{*}$, we have for $s=0,1,2,3$,

$$
\begin{aligned}
\left\|\nabla^{s} \varrho_{l}(t)\right\|_{L^{2}} & \geq c(1+t)^{-\left(\frac{3}{4}+\frac{s}{2}\right)} ; \\
\left\|\nabla^{s} m_{l}(t)\right\|_{L^{2}} & \geq c(1+t)^{-\left(\frac{3}{4}+\frac{s}{2}\right)},
\end{aligned}
$$

where $c$ is a positive constant independent of time $t$.

Proof. By virtue of the semigroup theory for evolution equation, the solution $\left(\varrho_{l}, m_{l}\right)$ of the linearized problem (2.4), (2.5) can be expressed by

$$
U_{t}=B U, U(0)=U_{0},
$$

where $U=\left(\varrho_{l}, m_{l}\right)^{t}$ and

$$
B=\left[\begin{array}{ll}
0 & -\operatorname{div} \\
-\nabla+\kappa \nabla \Delta & \mu \Delta+(\mu+\nu) \nabla \operatorname{div}
\end{array}\right] .
$$

Applying Fourier transform to Eq. (2.7), it holds on

$$
\hat{U}_{t}=A(\xi) \hat{U}, \hat{U}(0)=\hat{U}_{0},
$$

where

$$
A(\xi)=\left[\begin{array}{ll}
0 & -i \xi^{t} \\
-i \xi-i \kappa|\xi|^{2} \xi & -\mu|\xi|^{2} I_{3 \times 3}-(\mu+\nu) \xi \xi^{t}
\end{array}\right] .
$$

Then by computation, we obtain the Fourier transform $\hat{G}(\xi, t)$ of Green's function $G(x, t)=e^{t B}$ as follows:

$$
\hat{G}(\xi, t)=\left[\begin{array}{ll}
\frac{\lambda_{+} e^{\lambda_{-} t}-\lambda_{-} e^{\lambda_{+} t}}{\lambda_{+}-\lambda_{-}} & -\frac{i \xi^{t}\left(e^{\lambda_{+} t}-e^{\lambda_{-}}{ }^{\lambda_{+}}\right)}{\lambda_{+}-\lambda_{-}} \\
-\frac{i \xi\left(1+\kappa|\xi|^{2}\right)\left(e^{\lambda_{+}}-e^{\lambda_{-} t}\right)}{\lambda_{+}-\lambda_{-}} & \frac{\lambda_{+} e^{\lambda_{+}}-\lambda_{-} e^{\lambda_{-}}}{\lambda_{+}-\lambda_{-}} \cdot \frac{\xi \xi^{t}}{|\xi|^{2}}+e^{-\lambda_{0} t}\left(I-\frac{\xi \xi^{t}}{|\xi|^{2}}\right)
\end{array}\right],
$$

where

$$
\begin{aligned}
& \lambda_{0}=-\mu|\xi|^{2} \text { (double), } \\
& \lambda_{+}=-\frac{1}{2}(2 \mu+\nu)|\xi|^{2}+\frac{1}{2} i \sqrt{4\left(1+\kappa|\xi|^{2}\right)|\xi|^{2}-(2 \mu+\nu)^{2}|\xi|^{4}} \\
& \lambda_{-}=-\frac{1}{2}(2 \mu+\nu)|\xi|^{2}-\frac{1}{2} i \sqrt{4\left(1+\kappa|\xi|^{2}\right)|\xi|^{2}-(2 \mu+\nu)^{2}|\xi|^{4}} .
\end{aligned}
$$

Thus, we can obtain the expression for $\hat{\varrho}_{l}(\xi, t)$ and $\hat{m}_{l}(\xi, t)$ as follows,

$$
\hat{\varrho}_{l}(\xi, t)=\frac{\lambda_{+} e^{\lambda_{-} t}-\lambda_{-} e^{\lambda_{+} t}}{\lambda_{+}-\lambda_{-}} \hat{\varrho}_{l_{0}}-\frac{i \xi^{t}\left(e^{\lambda_{+} t}-e^{\lambda_{-} t}\right)}{\lambda_{+}-\lambda_{-}} \hat{m}_{l_{0}},
$$


and

$$
\hat{m}_{l}(\xi, t)=-\frac{i \xi\left(1+\kappa|\xi|^{2}\right)\left(e^{\lambda_{+} t}-e^{\lambda_{-} t}\right)}{\lambda_{+}-\lambda_{-}} \hat{\varrho}_{l_{0}}+\left[\frac{\lambda_{+} e^{\lambda_{+} t}-\lambda_{-} e^{\lambda_{-} t}}{\lambda_{+}-\lambda_{-}} \cdot \frac{\xi \xi^{t}}{|\xi|^{2}}+e^{-\lambda_{0} t}\left(I-\frac{\xi \xi^{t}}{|\xi|^{2}}\right)\right] \hat{m}_{l_{0}} .
$$

Then it is easy to verify that

$$
\hat{\varrho}_{l}(\xi, t) \sim\left\{\begin{array}{l}
e^{-\frac{1}{2}(2 \mu+\nu)|\xi|^{2} t} \cos (b t)\left|\hat{\varrho}_{l_{0}}\right|+\mathcal{O}(1) e^{-\frac{1}{2}(2 \mu+\nu)|\xi|^{2} t} \frac{\sin (b t)}{b}|\xi|^{2}\left|\hat{\varrho}_{l_{0}}\right|,|\xi| \ll 1, \\
\mathcal{O}(1) e^{-R_{0}|\xi|^{2} t}\left|\hat{\varrho}_{l_{0}}\right|,|\xi| \gg 1,
\end{array}\right.
$$

and

where

$$
\hat{m}_{l}(\xi, t) \sim\left\{\begin{array}{l}
e^{-\frac{1}{2}(2 \mu+\nu)|\xi|^{2} t} \sin (b t)\left|\hat{\varrho}_{l_{0}}\right|,|\xi| \ll 1 \\
\mathcal{O}(1) e^{-R_{0}|\xi|^{2} t}\left|\varrho_{l_{0}}\right|,|\xi| \gg 1
\end{array}\right.
$$

$$
b=\frac{1}{2} \sqrt{4\left(1+\kappa|\xi|^{2}\right)|\xi|^{2}-(2 \mu+\nu)^{2}|\xi|^{4}} \sim|\xi|+\mathcal{O}\left(|\xi|^{3}\right),|\xi| \ll 1,
$$

$R_{0}$ is a positive constant. By Parseval's theorem, for $s=0,1,2,3$, it holds on

$$
\begin{aligned}
\left\|\nabla^{s} \varrho_{l}\right\|_{L^{2}}^{2}= & \int_{|\xi| \leq \eta}|\xi|^{2 s}\left|\hat{\varrho}_{l}\right|^{2} \mathrm{~d} \xi+\int_{|\xi| \geq \eta}|\xi|^{2 s}\left|\hat{\varrho}_{l}\right|^{2} \mathrm{~d} \xi \\
\geq & \frac{1}{2} \int_{|\xi| \leq \eta} e^{-(2 \mu+\nu)|\xi|^{2} t}|\xi|^{2 s} \cos ^{2}(b t)\left|\hat{\varrho}_{l_{0}}\right|^{2} \mathrm{~d} \xi-C \int_{|\xi| \leq \eta} e^{-(2 \mu+\nu)|\xi|^{2} t}|\xi|^{2 s+4} \frac{\sin ^{2}(b t)}{b^{2}}\left|\hat{\varrho}_{l_{0}}\right|^{2} \mathrm{~d} \xi \\
& -C e^{-2 R_{0} \eta^{2} t},
\end{aligned}
$$

where $\eta$ is a small but fixed positive constant. Due to the fact that $b \sim|\xi|+\mathcal{O}\left(|\xi|^{3}\right)$ as $|\xi| \ll 1$, it holds on

$$
\int_{|\xi| \leq \eta} e^{-(2 \mu+\nu)|\xi|^{2} t}|\xi|^{2 s+4} \frac{\sin ^{2}(b t)}{b^{2}}\left|\hat{\varrho}_{l}\right|^{2} \mathrm{~d} \xi \leq C t^{-\left(s+\frac{5}{2}\right)} .
$$

Employing the mean value formula, we get

$$
\cos \left(|\xi|+\mathcal{O}\left(|\xi|^{3}\right)\right)=\cos (|\xi| t)+\mathcal{O}\left(|\xi|^{3} t\right)
$$

then, we have

$$
\cos ^{2}\left(|\xi|+\mathcal{O}\left(|\xi|^{3}\right)\right) \geq \frac{1}{2} \cos ^{2}(|\xi| t)-\mathcal{O}\left(\left[|\xi|^{3} t\right]^{2}\right)
$$

which, together with the above inequality, we obtain that

$$
\begin{aligned}
& \int_{|\xi| \leq \eta} e^{-(2 \mu+\nu)|\xi|^{2} t}|\xi|^{2 s} \cos ^{2}(b t)\left|\hat{\varrho}_{l_{0}}\right|^{2} \mathrm{~d} \xi \\
& \quad \geq C \int_{|\xi| \leq \eta} e^{-(2 \mu+\nu)|\xi|^{2} t}|\xi|^{2 s} \cos ^{2}(|\xi| t) \mathrm{d} \xi-C \int_{|\xi| \leq \eta} e^{-(2 \mu+\nu)|\xi|^{2} t}|\xi|^{2 s}|\xi|^{6} t^{2} \mathrm{~d} \xi \\
& \quad=: I_{1}-I_{2} .
\end{aligned}
$$

By direct computation, we have

$$
\int_{|\xi| \leq \eta} e^{-(2 \mu+\nu)|\xi|^{2} t}|\xi|^{2 s}|\xi|^{6} t^{2} \mathrm{~d} \xi \leq C t^{-\left(s+\frac{5}{2}\right)}
$$


therefore, we only need to consider the lower bound of $I_{1}$. We claim that the following estimate (which will be proved in Sect. 3),

$$
I_{1} \geq C t^{-\left(\frac{3}{2}+s\right)}
$$

Substituting the estimate (2.17) and (2.18) into (2.16), one arrives at

$$
\int_{|\xi| \leq \eta} e^{-(2 \mu+\nu)|\xi|^{2} t}|\xi|^{2 s} \cos ^{2}(b t)\left|\hat{\varrho}_{0}\right|^{2} \mathrm{~d} \xi \geq C t^{-\left(\frac{3}{2}+s\right)}
$$

Hence, we can easily obtain that for $s=0,1,2,3$,

$$
\left\|\nabla^{s} \varrho_{l}\right\|_{L^{2}}^{2} \geq C(1+t)^{-\left(\frac{3}{2}+s\right)}
$$

where $C$ is a positive constant independent of time. Similarly, we can also derive that for $s=0,1,2,3$,

$$
\left\|\nabla^{s} m_{l}\right\|_{L^{2}}^{2} \geq C(1+t)^{-\left(\frac{3}{2}+s\right)},
$$

with $C$ a positive constant independent of time. Therefore, we finish the proof of this proposition.

In order to obtain the lower bound for the solution of the compressible Navier-Stokes-Korteweg equation (2.1), we need to address the upper decay rate for the difference between the nonlinear and linearized part. Hence, let us denote

$$
\varrho_{\delta}:=\varrho-\varrho_{l}, m_{\delta}:=m-m_{l},
$$

then they satisfy the following system

$$
\left\{\begin{array}{l}
\partial_{t} \varrho_{\delta}+\operatorname{div} m_{\delta}=0 \\
\partial_{t} m_{\delta}-\mu \Delta m_{\delta}-(\mu+\nu) \nabla \operatorname{div} m_{\delta}+\nabla \varrho_{\delta}-\kappa \nabla \Delta \varrho_{\delta}=-\operatorname{div} S
\end{array}\right.
$$

with the zero initial data

$$
\left.\left(\varrho_{\delta}, m_{\delta}\right)(x, t)\right|_{t=0}=(0,0) .
$$

Here, the force term is defined in (2.2). Now we will establish the decay rate for the solution $\left(\varrho_{\delta}, m_{\delta}\right)$ of equation (2.19) in the following.

Lemma 2.2. For any smooth solution $\left(\varrho_{\delta}, m_{\delta}\right)$ of the equation $(2.19)$, it holds on

$$
\begin{aligned}
& \frac{\mathrm{d}}{\mathrm{d} t}\left(\left\|\nabla^{l}\left(\varrho \delta, m_{\delta}\right)\right\|_{H^{3-l}}^{2}+\kappa\left\|\nabla^{l+1} \varrho\right\|_{H^{3-l}}^{2}\right)+C\left\|\nabla^{l+1} m_{\delta}\right\|_{H^{3-l}}^{2} \\
& \quad \leq C\left\|\nabla^{l}(\varrho, u)\right\|_{H^{3-l}}^{2}\left(\|\nabla \varrho\|_{H^{2}}^{2}+\|\nabla u\|_{H^{2}}^{2}\right)+C\|\nabla \varrho\|_{H^{1}}^{2}\left(\left\|\nabla^{4} \varrho\right\|_{H^{1}}^{2}+\left\|\nabla^{4} u\right\|_{L^{2}}^{2}\right),
\end{aligned}
$$

and

$$
\begin{aligned}
& \sum_{k=l}^{3} \frac{\mathrm{d}}{\mathrm{d} t} \int \nabla^{l} m_{\delta} \cdot \nabla^{l+1} \varrho_{\delta} \mathrm{d} x+\left\|\nabla^{l+1} \varrho_{\delta}\right\|_{H^{3-l}}^{2}+\frac{1}{2} \kappa\left\|\nabla^{l+2} \varrho_{\delta}\right\|_{H^{3-l}}^{2} \\
& \leq C\left\|\nabla^{l+1} m_{\delta}\right\|_{H^{3-l}}^{2}+C\left\|\nabla^{l}(\varrho, u)\right\|_{H^{3-l}}^{2}\left(\|\nabla \varrho\|_{H^{2}}^{2}+\|\nabla u\|_{H^{2}}^{2}\right) \\
& \quad+C\|\nabla \varrho\|_{H^{1}}^{2}\left(\left\|\nabla^{4} \varrho\right\|_{H^{1}}^{2}+\left\|\nabla^{4} u\right\|_{L^{2}}^{2}\right)
\end{aligned}
$$

where $l=0,1,2,3$.

The above inequalities (2.21) and (2.22) in Lemma 2.2 will be proved later in Sect. 3. Multiplying inequality (2.22) by a small constant $\delta$ and adding with (2.21), together with the decay rates (1.7), we get for all $t \geq 0$,

$$
\begin{aligned}
& \frac{\mathrm{d}}{\mathrm{d} t} \mathcal{E}_{l}^{3}(t)+\frac{\delta}{2}\left\|\nabla^{l+1} \varrho_{\delta}\right\|_{H^{3-l}}^{2}+\frac{\delta}{2} \kappa\left\|\nabla^{l+2} \varrho_{\delta}\right\|_{H^{3-l}}^{2}+C\left\|\nabla^{l+1} m_{\delta}\right\|_{H^{3-l}}^{2} \\
& \quad \leq C(1+t)^{-(4+l)}+C(1+t)^{-\frac{5}{2}}\left(\left\|\nabla^{4} \varrho\right\|_{H^{1}}^{2}+\left\|\nabla^{4} u\right\|_{L^{2}}^{2}\right),
\end{aligned}
$$


where $l=0,1,2,3$. Here, the energy $\mathcal{E}_{l}^{3}(t)$ is defined by

$$
\mathcal{E}_{l}^{3}(t):=\left\|\nabla^{l}\left(\varrho_{\delta}, m_{\delta}\right)\right\|_{H^{3-l}}^{2}+\kappa\left\|\nabla^{l+1} \varrho_{\delta}\right\|_{H^{3-l}}^{2}+\delta \sum_{k=l}^{3} \int \nabla^{k} m_{\delta} \cdot \nabla^{k+1} \varrho_{\delta} \mathrm{d} x .
$$

Due to the smallness of $\delta$, there are two constants $C_{*}$ and $C^{*}$ (independent of time) such that

$$
C_{*}\left(\left\|\nabla^{l} \varrho_{\delta}(t)\right\|_{H^{4-l}}^{2}+\left\|\nabla^{l} m_{\delta}(t)\right\|_{H^{3-l}}^{2}\right) \leq \mathcal{E}_{l}^{3}(t) \leq C^{*}\left(\left\|\nabla^{l} \varrho_{\delta}(t)\right\|_{H^{4-l}}^{2}+\left\|\nabla^{l} m_{\delta}(t)\right\|_{H^{3-l}}^{2}\right) .
$$

Now we establish the upper bound decay rate of solution $\left(\varrho_{\delta}, m_{\delta}\right)$ for the equation (2.19).

Lemma 2.3. Under the assumptions in Theorem 1.1, the smooth solution $\left(\varrho_{\delta}, m_{\delta}\right)$ of equation satisfies

$$
\left\|\nabla^{l} \varrho_{\delta}(t)\right\|_{H^{4-l}}+\left\|\nabla^{l} m_{\delta}(t)\right\|_{H^{3-l}} \leq C(1+t)^{-\frac{5+2 l}{4}},
$$

where $l=0,1,2,3$. Here, $C$ is a constant independent of time.

Proof. We will take the strategy of induction to give the proof of estimate (2.26). Taking $l=0$ in (2.23), then we have

$$
\frac{\mathrm{d}}{\mathrm{d} t} \mathcal{E}_{0}^{3}(t)+C\left(\left\|\nabla \varrho_{\delta}\right\|_{H^{4}}^{2}+\left\|\nabla m_{\delta}\right\|_{H^{3}}^{2}\right) \leq C(1+t)^{-4}+C(1+t)^{-\frac{5}{2}}\left(\left\|\nabla^{4} \varrho\right\|_{H^{1}}^{2}+\left\|\nabla^{4} u\right\|_{L^{2}}^{2}\right) .
$$

Obviously, the dissipation term $\left\|\nabla \varrho_{\delta}\right\|_{H^{4}}^{2}+\left\|\nabla m_{\delta}\right\|_{H^{3}}^{2}$ cannot control the energy term $\mathcal{E}_{0}^{3}(t)$ in above inequality. Thus, we add both sides of the above inequality with term $\left\|\left(\varrho_{\delta}, m_{\delta}\right)\right\|_{L^{2}}^{2}$ and get

$$
\begin{aligned}
& \frac{\mathrm{d}}{\mathrm{d} t} \mathcal{E}_{0}^{3}(t)+C\left(\left\|\varrho_{\delta}\right\|_{H^{5}}^{2}+\left\|m_{\delta}\right\|_{H^{4}}^{2}\right) \\
& \quad \leq\left\|\left(\varrho_{\delta}, m_{\delta}\right)\right\|_{L^{2}}^{2}+C(1+t)^{-4}+C(1+t)^{-\frac{5}{2}}\left(\left\|\nabla^{4} \varrho\right\|_{H^{1}}^{2}+\left\|\nabla^{4} u\right\|_{L^{2}}^{2}\right) .
\end{aligned}
$$

By virtue of the Duhamel principle formula and estimate (1.7), we have

$$
\begin{aligned}
\left\|\left(\varrho_{\delta}, m_{\delta}\right)(t)\right\|_{L^{2}} & \leq \int_{0}^{t}(1+t-\tau)^{-\frac{5}{4}}\left(\left\||\xi|^{-1} \mathcal{F}(\operatorname{div} S)\right\|_{L^{\infty}}+\|\operatorname{div} S\|_{L^{2}}\right) \mathrm{d} \tau \\
& \leq \int_{0}^{t}(1+t-\tau)^{-\frac{5}{4}}\left(\|S\|_{L^{1}}+\|\operatorname{div} S\|_{L^{2}}\right) \mathrm{d} \tau \\
& \leq C \int_{0}^{t}(1+t-\tau)^{-\frac{5}{4}}(1+\tau)^{-\frac{3}{2}} \mathrm{~d} \tau \\
& \leq C(1+t)^{-\frac{5}{4}}
\end{aligned}
$$

where we have used Sobolev inequality to get

$$
\begin{aligned}
& \|S\|_{L^{1}}+\|\operatorname{div} S\|_{L^{2}} \\
& \leq\|\varrho+1\|_{L^{\infty}}\|u\|_{L^{2}}\|u\|_{L^{2}}+\|\nabla \varrho\|_{L^{2}}\|u\|_{L^{2}}+\|\varrho\|_{L^{2}}\|\nabla u\|_{L^{2}}+\|\varrho\|_{L^{2}}\|\varrho\|_{L^{2}} \\
& \quad+\|\varrho\|_{L^{2}}\left\|\nabla^{2} \varrho\right\|_{L^{2}}+\|\nabla \varrho\|_{L^{2}}\|\nabla \varrho\|_{L^{2}}+\|1+\varrho\|_{L^{\infty}}\|u\|_{L^{\infty}}\|\nabla u\|_{L^{2}}+\|u\|_{L^{\infty}}^{2}\|\nabla \varrho\|_{L^{2}} \\
& \quad+\left\|\nabla^{2}(\varrho u)\right\|_{L^{2}}+\|\nabla \operatorname{div}(\varrho u)\|_{L^{2}}+\left\|\nabla\left(\varrho \nabla^{2} \varrho\right)\right\|_{L^{2}}+\|\varrho \nabla \varrho\|_{L^{2}} \\
& \leq C\|\nabla(\varrho, u)\|_{H^{1}}\|(\varrho, u)\|_{L^{2}}+C\|\varrho\|_{L^{2}}^{2}+C\|\nabla \varrho\|_{L^{2}}^{2}+C\|\nabla(\varrho, u)\|_{H^{1}}^{2}+C\|\nabla \varrho\|_{H^{1}}\left\|\nabla^{3} \varrho\right\|_{L^{2}} \\
& \leq \\
& \leq C(1+t)^{-\frac{3}{2}} .
\end{aligned}
$$

Using (2.27), (2.28) and equivalent relation (2.25), one arrives at

$$
\frac{\mathrm{d}}{\mathrm{d} t} \mathcal{E}_{0}^{3}(t)+\frac{C}{C^{*}} \mathcal{E}_{0}^{3}(t) \leq C(1+t)^{-\frac{5}{2}}+C(1+t)^{-\frac{5}{2}}\left(\left\|\nabla^{4} \varrho\right\|_{H^{1}}^{2}+\left\|\nabla^{4} u\right\|_{L^{2}}^{2}\right),
$$


which implies

$$
\mathcal{E}_{0}^{3}(t) \leq C \int_{0}^{t} e^{-\frac{C}{C^{*}}(t-\tau)}(1+\tau)^{-\frac{5}{2}} \mathrm{~d} \tau+C \int_{0}^{t} e^{-\frac{C}{C^{*}}(t-\tau)}(1+\tau)^{-\frac{5}{2}}\left(\left\|\nabla^{4} \varrho\right\|_{H^{1}}^{2}+\left\|\nabla^{4} u\right\|_{L^{2}}^{2}\right) \mathrm{d} \tau .
$$

It is easy to obtain that

$$
\int_{0}^{t} e^{-\frac{C}{C^{*}}(t-\tau)}(1+\tau)^{-\frac{5}{2}} \mathrm{~d} \tau \leq C(1+t)^{-\frac{5}{2}}
$$

where we have used the fact that

$$
e^{-\frac{C}{C^{*}} t} \leq C(1+t)^{-\frac{5}{2}}
$$

for some constant $C>0$, and

$$
\begin{aligned}
& \int_{0}^{t} e^{-\frac{C}{C^{*}}(t-\tau)}(1+\tau)^{-\frac{5}{2}} \mathrm{~d} \tau \\
& \leq C \int_{0}^{t}(1+t-\tau)^{-\frac{5}{2}}(1+\tau)^{-\frac{5}{2}} \mathrm{~d} \tau \\
& \quad=C \int_{0}^{\frac{t}{2}}+\int_{\frac{t}{2}}^{t}(1+t-\tau)^{-\frac{5}{2}}(1+\tau)^{-\frac{5}{2}} \mathrm{~d} \tau \\
& \leq C\left(1+\frac{t}{2}\right)^{-\frac{5}{2}} \int_{0}^{\frac{t}{2}}(1+\tau)^{-\frac{5}{2}} \mathrm{~d} \tau+\left(1+\frac{t}{2}\right)^{-\frac{5}{2}} \int_{\frac{t}{2}}^{t}(1+t-\tau)^{-\frac{5}{2}} \mathrm{~d} \tau \\
& \leq C(1+t)^{-\frac{5}{2}} .
\end{aligned}
$$

In the sequel, we only need to deal with the term

$$
\int_{0}^{t} e^{-\frac{C}{C^{*}}(t-\tau)}(1+\tau)^{-\frac{5}{2}}\left(\left\|\nabla^{4} \varrho\right\|_{H^{1}}^{2}+\left\|\nabla^{4} u\right\|_{L^{2}}^{2}\right) \mathrm{d} \tau .
$$

We claim the estimate(which will be proved in Sect. 3),

$$
\int_{0}^{t} e^{-\frac{C}{C^{*}}(t-\tau)}(1+\tau)^{-\frac{5}{2}}\left(\left\|\nabla^{4} \varrho\right\|_{H^{1}}^{2}+\left\|\nabla^{4} u\right\|_{L^{2}}^{2}\right) \mathrm{d} \tau \leq C(1+t)^{-\frac{5}{2}} .
$$

Hence, we can easily obtain that

$$
\left\|\varrho_{\delta}(t)\right\|_{H^{4}}^{2}+\left\|m_{\delta}(t)\right\|_{H^{3}}^{2} \leq C(1+t)^{-\frac{5}{2}} .
$$

We now assume that the decay rate (2.26) holds on for the case $k=l$, i.e.,

$$
\left\|\nabla^{l} \varrho_{\delta}(t)\right\|_{H^{4-l}}+\left\|\nabla^{l} m_{\delta}(t)\right\|_{H^{3-l}} \leq C(1+t)^{-\frac{5+2 l}{4}},
$$

for $l=1,2$. Then, we should verify that the estimate $(2.26)$ holds on for the case $k=l+1$. Indeed, we replace $l$ by $l+1$ in (2.23) to obtain that

$$
\begin{aligned}
& \frac{\mathrm{d}}{\mathrm{d} t} \mathcal{E}_{l+1}^{3}(t)+C\left(\left\|\nabla^{l+2} \varrho_{\delta}\right\|_{H^{3-l}}^{2}+\left\|\nabla^{l+2} m_{\delta}\right\|_{H^{2-l}}^{2}\right) \\
& \quad \leq C(1+t)^{-(5+l)}+C(1+t)^{-\frac{5}{2}}\left(\left\|\nabla^{4} \varrho\right\|_{H^{1}}^{2}+\left\|\nabla^{4} u\right\|_{L^{2}}^{2}\right) .
\end{aligned}
$$


For some constant $R$ defined below, denoting the time sphere (see [19])

$$
S_{0}:=\left\{\xi \in \mathbb{R}^{3}|| \xi \mid \leq\left(\frac{R}{1+t}\right)^{\frac{1}{2}}\right\}
$$

it follows immediately

$$
\begin{gathered}
\left\|\nabla^{l+2} \varrho_{\delta}\right\|_{H^{3-l}}^{2} \geq \frac{R}{1+t}\left\|\nabla^{l+1} \varrho_{\delta}\right\|_{H^{3-l}}^{2}-\frac{R^{2}}{(1+t)^{2}}\left\|\nabla^{l} \varrho_{\delta}\right\|_{H^{3-l}}^{2} \\
\left\|\nabla^{l+2} m_{\delta}\right\|_{H^{2-l}}^{2} \geq \frac{R}{1+t}\left\|\nabla^{l+1} m_{\delta}\right\|_{H^{2-l}}^{2}-\frac{R^{2}}{(1+t)^{2}}\left\|\nabla^{l} m_{\delta}\right\|_{H^{2-l}}^{2} .
\end{gathered}
$$

By substituting (2.34) into (2.33), we can easily get

$$
\begin{aligned}
& \frac{\mathrm{d}}{\mathrm{d} t} \mathcal{E}_{l+1}^{3}(t)+\frac{C R}{1+t}\left(\left\|\nabla^{l+1} \varrho_{\delta}\right\|_{H^{3-l}}^{2}+\left\|\nabla^{l+1} m_{\delta}\right\|_{H^{2-l}}^{2}\right) \\
& \quad \leq \frac{C R^{2}}{(1+t)^{2}}\left(\left\|\nabla^{l} \varrho_{\delta}\right\|_{H^{3-l}}^{2}+\left\|\nabla^{l} m_{\delta}\right\|_{H^{2-l}}^{2}\right)+C(1+t)^{-(5+l)}+C(1+t)^{-\frac{5}{2}}\left(\left\|\nabla^{4} \varrho\right\|_{H^{1}}^{2}+\left\|\nabla^{4} u\right\|_{L^{2}}^{2}\right) \\
& \quad \leq C R^{2}(1+t)^{-\frac{9+2 l}{2}}+C(1+t)^{-(5+l)}+C(1+t)^{-\frac{5}{2}}\left(\left\|\nabla^{4} \varrho\right\|_{H^{1}}^{2}+\left\|\nabla^{4} u\right\|_{L^{2}}^{2}\right),
\end{aligned}
$$

where we have used the assumption (2.32). Notice that the term $\mathcal{E}_{l+1}^{3}(t)$ is equivalent to the norm $\left\|\nabla^{l+1} \varrho_{\delta}\right\|_{H^{4-l}}^{2}+\left\|\nabla^{l+1} m_{\delta}\right\|_{H^{3-l}}^{2}$; hence, we obtain

$$
\begin{aligned}
& \frac{\mathrm{d}}{\mathrm{d} t} \mathcal{E}_{l+1}^{3}(t)+\frac{C R}{C^{*}(1+t)} \mathcal{E}_{l+1}^{3}(t) \\
& \quad \leq C R^{2}(1+t)^{-\frac{9+2 l}{2}}+C(1+t)^{-(5+l)}+C(1+t)^{-\frac{5}{2}}\left(\left\|\nabla^{4} \varrho\right\|_{H^{1}}^{2}+\left\|\nabla^{4} u\right\|_{L^{2}}^{2}\right) .
\end{aligned}
$$

Choosing $R=C^{*}(l+4) / C$ and multiplying the above inequality by $(1+t)^{l+4}$, we obtain that

$$
\frac{\mathrm{d}}{\mathrm{d} t}\left[(1+t)^{l+4} \mathcal{E}_{l+1}^{3}(t)\right] \leq C(1+t)^{-\frac{1}{2}}+C(1+t)^{l+\frac{3}{2}}\left(\left\|\nabla^{4} \varrho\right\|_{H^{1}}^{2}+\left\|\nabla^{4} u\right\|_{L^{2}}^{2}\right) .
$$

We claim that the following estimate holds on (which will be proved in Sect. 3),

$$
\int_{0}^{t}(1+\tau)^{l+\frac{3}{2}}\left(\left\|\nabla^{4} \varrho(\tau)\right\|_{H^{1}}^{2}+\left\|\nabla^{4} u(\tau)\right\|_{L^{2}}^{2}\right) \mathrm{d} \tau \leq C,
$$

which together with (2.35) yields directly

$$
\mathcal{E}_{l+1}^{3}(t) \leq C(1+t)^{-\frac{7+2 l}{2}} .
$$

Then, due to the fact that the term $\mathcal{E}_{l+1}^{3}(t)$ is equivalent to the norm $\left\|\nabla^{l+1} \varrho_{\delta}\right\|_{H^{4-l}}^{2}+\left\|\nabla^{l+1} m_{\delta}\right\|_{H^{3-l}}^{2}$, we obtain

$$
\left\|\nabla^{l+1} \varrho_{\delta}\right\|_{H^{4-l}}^{2}+\left\|\nabla^{l+1} m_{\delta}\right\|_{H^{3-l}}^{2} \leq C(1+t)^{-\frac{7+2 l}{2}} .
$$

Thus, by the general step of induction, we have given the proof for (2.26).

Finally, we establish the lower bound estimates.

Lemma 2.4. Under all the assumptions of Theorem 1.2, the solution $(\rho, u)$ of equation (1.1) has the following estimate

$$
\begin{aligned}
& \left\|\nabla^{k}(\rho-1)(t)\right\|_{L^{2}} \geq C(1+t)^{-\frac{3+2 k}{4}}, \quad k=0,1,2,3 \\
& \left\|\nabla^{k} u(t)\right\|_{L^{2}} \geq C(1+t)^{-\frac{3+2 k}{4}}, \quad k=0,1,2,3
\end{aligned}
$$

for all $t \geq t_{*}$, where $t_{*}$ is a positive constant. 
Proof. Recall the definition

$$
\varrho_{\delta}=\varrho-\varrho_{l}, m_{\delta}=m-m_{l},
$$

we have

$$
\left\|\varrho_{l}\right\|_{L^{2}}=\left\|\varrho-\varrho_{\delta}\right\|_{L^{2}} \leq\|\varrho\|_{L^{2}}+\|\varrho \delta\|_{L^{2}},
$$

which, together with estimates (2.6) and (2.26), yields directly

$$
\begin{aligned}
\|\varrho(t)\|_{L^{2}} & \geq\left\|\varrho_{l}(t)\right\|_{L^{2}}-\left\|\varrho_{\delta}(t)\right\|_{L^{2}} \\
& \geq C_{1}(1+t)^{-\frac{3}{4}}-C_{2}(1+t)^{-\frac{5}{4}} \\
& \geq C_{1}(1+t)^{-\frac{3}{4}}-\frac{C_{2}}{(1+t)^{\frac{1}{2}}}(1+t)^{-\frac{3}{4}} .
\end{aligned}
$$

Choosing $t \geq \frac{4 C_{2}^{2}-C_{1}^{2}}{C_{1}^{2}}$, it holds on

$$
\|\varrho(t)\|_{L^{2}} \geq C(1+t)^{-\frac{3}{4}} .
$$

Similarly, using estimates (2.6) and (2.26), we also have

$$
\begin{aligned}
&\left\|\nabla^{k} \varrho(t)\right\|_{L^{2}} \geq C(1+t)^{-\frac{3+2 k}{4}}, \quad k=1,2,3 ; \\
&\left\|\nabla^{k} m(t)\right\|_{L^{2}} \geq C(1+t)^{-\frac{3+2 k_{1}}{4}}, \quad k=0,1,2,3 .
\end{aligned}
$$

Finally, we establish the lower decay rate for the velocity. Using decay estimate (1.7) and Morse inequality, we get

$$
\begin{aligned}
\left\|\nabla^{k} m\right\|_{L^{2}} & \leq\left\|\nabla^{k} u\right\|_{L^{2}}+\left\|\nabla^{k}(\varrho u)\right\|_{L^{2}} \\
& \leq\left\|\nabla^{k} u\right\|_{L^{2}}+C\|\varrho\|_{L^{\infty}}\left\|\nabla^{k} u\right\|_{L^{2}}+C\|u\|_{L^{\infty}}\left\|\nabla^{k} \varrho\right\|_{L^{2}} \\
& \leq\left\|\nabla^{k} u\right\|_{L^{2}}+C(1+t)^{-\frac{9+2 k}{4}},
\end{aligned}
$$

which, together with (2.38), yields directly

$$
\left\|\nabla^{k} u\right\|_{L^{2}} \geq\left\|\nabla^{k} m\right\|_{L^{2}}-C(1+t)^{-\frac{9+2 k}{4}} \geq C(1+t)^{-\frac{3+2 k}{4}} .
$$

Therefore, we complete the proof of lemma.

\subsection{Upper and lower bounds of decay for time derivative}

In this subsection, we will establish the upper and lower bounds for the time derivatives of density and velocity. In [25], Wang and Tan have rewriten (1.1) in the perturbation form as

$$
\left\{\begin{array}{l}
\varrho_{t}+\operatorname{div} u=G_{1}, \\
u_{t}-\mu \Delta u-(\mu+\nu) \nabla \operatorname{div} u+\nabla \varrho-\kappa \nabla \Delta \varrho=G_{2},
\end{array}\right.
$$

where $\varrho=\rho-1$, and the function $G_{i}(i=1,2)$ is defined as

$$
\left\{\begin{array}{l}
G_{1}=-\varrho \operatorname{div} u-u \cdot \nabla \varrho \\
G_{2}=-u \cdot \nabla u-\frac{\varrho}{\varrho+1}(\mu \Delta u+(\mu+\nu) \nabla \operatorname{div} u)-\left(\frac{P^{\prime}(\varrho+1)}{\varrho+1}-1\right) \nabla \varrho .
\end{array}\right.
$$

The initial data are given as

$$
\left.(\varrho, u)(x, t)\right|_{t=0}=\left(\varrho_{0}, u_{0}\right)(x) \rightarrow(0,0) \quad \text { as } \quad|x| \rightarrow \infty .
$$

Now, we establish the upper and lower bounds of decay rate for the time derivative of solution in the $L^{2}$ norm. The lower decay rate estimate for the time derivative of density and velocity can be obtained by using the method in [9]. However, we still give the estimate in detail due to the appearance of the Korteweg term. 
Lemma 2.5. Under the assumptions in Theorem 1.2, the global solution ( $\varrho, u)$ of equation (2.39) has the following estimate

$$
\begin{aligned}
& C(1+t)^{-\frac{5}{4}} \leq\left\|\partial_{t} \varrho(t)\right\|_{L^{2}} \leq C(1+t)^{-\frac{5}{4}} \\
& C(1+t)^{-\frac{5}{4}} \leq\left\|\partial_{t} u(t)\right\|_{L^{2}} \leq C(1+t)^{-\frac{5}{4}},
\end{aligned}
$$

for all $t \geq t_{*}$ with $t_{*}$ a positive constant. Here, $C$ is a positive constant independent of time.

Proof. At first, we establish upper bound time decay rate for $\partial_{t} \varrho$ and $\partial_{t} u$ in the $L^{2}$ norm. With the help of the equation (2.39), we can easily obtain

$$
\left\|\partial_{t} \varrho\right\|_{L^{2}} \leq C\|\operatorname{div} u\|_{L^{2}}+\left\|G_{1}\right\|_{L^{2}}
$$

and

$$
\left\|\partial_{t} u\right\|_{L^{2}} \leq C\|\Delta u\|_{L^{2}}+C\|\nabla \operatorname{div} u\|_{L^{2}}+C\|\nabla \varrho\|_{L^{2}}+C\|\nabla \Delta \varrho\|_{L^{2}}+\left\|G_{2}\right\|_{L^{2}} .
$$

By virtue of Sobolev's inequality and time decay rate (1.7), we have

$$
\begin{aligned}
\left\|G_{1}\right\|_{L^{2}} & \leq\|\varrho \operatorname{div} u\|_{L^{2}}+\|u \cdot \nabla \varrho\|_{L^{2}} \\
& \leq C\|\varrho\|_{L^{\infty}}\|\operatorname{div} u\|_{L^{2}}+C\|u\|_{L^{\infty}}\|\nabla \varrho\|_{L^{2}} \\
& \leq C(1+t)^{-\frac{5}{2}}
\end{aligned}
$$

and

$$
\begin{aligned}
\left\|G_{2}\right\|_{L^{2}} \leq & C\|u \cdot \nabla u\|_{L^{2}}+C\left\|\frac{\varrho}{1+\varrho} \Delta u\right\|_{L^{2}}+C\left\|\frac{\varrho}{1+\varrho} \nabla \operatorname{div} u\right\|_{L^{2}}+C\left\|\left(\frac{P^{\prime}(1+\varrho)}{1+\varrho}-1\right) \nabla \varrho\right\|_{L^{2}} \\
\leq & \|u\|_{L^{\infty}}\|\nabla u\|_{L^{2}}+\left\|\frac{1}{1+\varrho}\right\|_{L^{\infty}}\|\varrho\|_{L^{\infty}}\left\|\nabla^{2} u\right\|_{L^{2}}+\left\|\frac{1}{1+\varrho}\right\|_{L^{\infty}}\|\nabla \varrho\|_{L^{6}}\|\nabla \varrho\|_{L^{3}} \\
& +\left\|\frac{1}{1+\varrho}\right\|_{L^{\infty}}\|\varrho\|_{L^{\infty}}\|\nabla \varrho\|_{L^{2}} \\
\leq & C(1+t)^{-\frac{5}{2}}
\end{aligned}
$$

Then, we can easily derive that

$$
\left\|\partial_{t} \varrho\right\|_{L^{2}} \leq C(1+t)^{-\frac{5}{4}}
$$

and

$$
\left\|\partial_{t} u\right\|_{L^{2}} \leq C(1+t)^{-\frac{5}{4}}
$$

Next, we establish lower bound time decay rate for $\partial_{t} u$ in the $L^{2}$ norm. Using the momentum equation in (2.39), we have

$$
\|\nabla \varrho\|_{L^{2}} \leq\left\|\partial_{t} u\right\|_{L^{2}}+C\left\|\nabla^{2} u\right\|_{L^{2}}+C\|\nabla \Delta \varrho\|_{L^{2}}+\left\|G_{2}\right\|_{L^{2}} .
$$

With the help of the inequality (2.43), we can get for all $t \geq t_{*}$,

$$
\left\|\partial_{t} u\right\|_{L^{2}} \geq C(1+t)^{-\frac{5}{4}}-C(1+t)^{-\frac{7}{4}}-C(1+t)^{-\frac{9}{4}}-C(1+t)^{-\frac{5}{2}} \geq C(1+t)^{-\frac{5}{4}} .
$$

Finally, we establish lower bound time decay rate for $\partial_{t} \varrho$ in the $L^{2}$ norm. To achieve this target, we use the transport equation in (2.39) to obtain

$$
\|\operatorname{div} u\|_{L^{2}} \leq\left\|\partial_{t} \varrho\right\|_{L^{2}}+\left\|G_{1}\right\|_{L^{2}} ;
$$

hence, together with the inequality (2.42), we obtain

$$
\left\|\partial_{t} \varrho\right\|_{L^{2}} \geq\|\operatorname{div} u\|_{L^{2}}-C(1+t)^{-\frac{5}{2}}
$$

Now, we need to establish the lower bound decay rate for $\|\operatorname{div} u\|_{L^{2}}$. Notice the differential relation $\Delta=\nabla \operatorname{div}-\nabla \times \nabla \times$, we get

$$
\|\nabla u\|_{L^{2}}^{2}=\|\operatorname{div} u\|_{L^{2}}^{2}+\|\nabla \times u\|_{L^{2}}^{2} .
$$


And hence, one arrives at

$$
\|\operatorname{div} u\|_{L^{2}} \geq C\|\nabla u\|_{L^{2}}-C\|\nabla \times u\|_{L^{2}} \geq C(1+t)^{-\frac{5}{4}}-C\|\nabla \times u\|_{L^{2}},
$$

which implies that we need to establish upper bound decay rate for $\|\nabla \times u\|_{L^{2}}$. To this end, applying the operator $\nabla \times$ to both sides of the equation (2.39), we arrive at

$$
\partial_{t}(\nabla \times u)-\mu \Delta(\nabla \times u)=\nabla \times G_{2} .
$$

Using Sobolev's inequality, uniform bound (1.6) and decay rate (1.7), we have

$$
\begin{aligned}
& \left\|G_{2}\right\|_{L^{1}}+\left\|G_{2}\right\|_{L^{2}} \\
& \leq C\|u \cdot \nabla u\|_{L^{1}}+C\left\|\frac{\varrho}{1+\varrho} \Delta u\right\|_{L^{1}}+C\left\|\frac{\varrho}{1+\varrho} \nabla \operatorname{div} u\right\|_{L^{1}}+C\left\|\left(\frac{P^{\prime}(1+\varrho)}{1+\varrho}-1\right) \nabla \varrho\right\|_{L^{1}} \\
& \quad+C\|u \cdot \nabla u\|_{L^{2}}+C\left\|\frac{\varrho}{1+\varrho} \Delta u\right\|_{L^{2}}+C\left\|\frac{\varrho}{1+\varrho} \nabla \operatorname{div} u\right\|_{L^{2}}+C\left\|\left(\frac{P^{\prime}(1+\varrho)}{1+\varrho}-1\right) \nabla \varrho\right\|_{L^{2}} \\
& \leq C\|u\|_{L^{2}}\|\nabla u\|_{L^{2}}+C\left\|\frac{\varrho}{1+\varrho}\right\|_{L^{\infty}}\|\varrho\|_{L^{2}}\left\|\nabla^{2} u\right\|_{L^{2}}+C\left\|\frac{\varrho}{1+\varrho}\right\|_{L^{\infty}}\|\varrho\|_{L^{2}}\left\|\nabla^{2} \varrho\right\|_{L^{2}} \\
& \quad+C\|u\|_{L^{3}}\|\nabla u\|_{L^{6}}+C\left\|\frac{\varrho}{1+\varrho}\right\|_{L^{\infty}}\|\varrho\|_{L^{3}}\left\|\nabla^{2} u\right\|_{L^{6}}+C\left\|\frac{\varrho}{1+\varrho}\right\|_{L^{\infty}}\|\varrho\|_{L^{3}}\left\|\nabla^{2} \varrho\right\|_{L^{6}} \\
& \leq C\|(\varrho, u)\|_{H^{1}}\|\nabla(\varrho, u)\|_{H^{2}} \\
& \leq C \delta(1+t)^{-\frac{5}{4}} .
\end{aligned}
$$

By virtue of the Duhamel principle formula and (2.47), we get

$$
\begin{aligned}
\|\nabla \times u\|_{L^{2}} \leq & C(1+t)^{-\frac{5}{4}}\left(\left\|\Lambda^{-1} \mathcal{F}\left(\nabla \times u_{0}\right)\right\|_{L^{\infty}}+\left\|\Lambda^{-1} \mathcal{F}\left(\nabla \times u_{0}\right)\right\|_{L^{2}}\right) \\
& +C \int_{0}^{t}(1+t-\tau)^{-\frac{5}{4}}\left(\left\|\Lambda^{-1} \mathcal{F}\left(\nabla \times G_{2}\right)\right\|_{L^{\infty}}+\left\|\Lambda^{-1} \mathcal{F}\left(\nabla \times G_{2}\right)\right\|_{L^{2}}\right) \mathrm{d} \tau \\
\leq & C(1+t)^{-\frac{5}{4}}\left(\left\|u_{0}\right\|_{L^{1}}+\left\|u_{0}\right\|_{L^{2}}\right)+C \int_{0}^{t}(1+t-\tau)^{-\frac{5}{4}}\left(\left\|G_{2}\right\|_{L^{1}}+\left\|G_{2}\right\|_{L^{2}}\right) \mathrm{d} \tau \\
\leq & C\left(\delta+\delta_{1}\right)(1+t)^{-\frac{5}{4}}+C \delta \int_{0}^{t}(1+t-\tau)^{-\frac{5}{4}}(1+\tau)^{-\frac{5}{4}} \mathrm{~d} \tau \\
\leq & C\left(\delta+\delta_{1}\right)(1+t)^{-\frac{5}{4}},
\end{aligned}
$$

which, together with estimates (2.45) and (2.46), yields directly

$$
\left\|\partial_{t} \varrho\right\|_{L^{2}} \geq C(1+t)^{-\frac{5}{4}}-C\left(\delta+\delta_{1}\right)(1+t)^{-\frac{5}{4}}-C(1+t)^{-\frac{5}{2}}
$$

where the positive constant $C$ on right-hand side of the above inequality depends on $c_{0}$ given in Proposition 2.1 , but not on $\delta$ and $\delta_{1}$. Then, by virtue of the smallness of $\delta$ and $\delta_{1}$, we have for $t \geq t_{*}$,

$$
\left\|\partial_{t} \varrho\right\|_{L^{2}} \geq C(1+t)^{-\frac{5}{4}} .
$$

Therefore, we complete the proof of this lemma.

\section{Proof of some technical estimates}

In this section, we will establish the claim estimates that have been used in Sect. 2. That is to say, we will establish the claim estimates (2.18), (2.21), (2.22), (2.30) and (2.36). 
Proof of inequality (2.18). Let $\zeta=\xi \sqrt{t}$, we obtain

$$
\begin{aligned}
& I_{1}=C \int_{|\xi| \leq \eta} e^{-(\mu+\nu)|\xi|^{2} t}|\xi|^{2 s} \cos ^{2}(|\xi| t) \mathrm{d} \xi \\
& =C t^{-\left(\frac{3}{2}+s\right)} \int_{|\zeta| \leq n t^{\frac{1}{2}}} e^{-(\mu+\nu)|\zeta|^{2}}|\zeta|^{2 s} \cos ^{2}\left(|\zeta| t^{\frac{1}{2}}\right) \mathrm{d} \zeta \\
& |\zeta| \leq \eta t^{\frac{1}{2}} \\
& =C t^{-\left(\frac{3}{2}+s\right)} \int_{0}^{\eta t^{\frac{1}{2}}} e^{-(\mu+\nu) R^{2}} R^{2 s+2} \cos ^{2}\left(R t^{\frac{1}{2}}\right) \mathrm{d} R \\
& \geq C t^{-\left(\frac{3}{2}+s\right)} \sum_{k=0}^{\left[\frac{\eta t}{\pi}\right]-1} \int_{\frac{k \pi+\frac{\pi}{6}}{\sqrt{t}}}^{\frac{k \pi+\frac{\pi}{3}}{\sqrt{t}}} e^{-(\mu+\nu) R^{2}} R^{2 s+2} \cos ^{2}\left(R t^{\frac{1}{2}}\right) \mathrm{d} R \\
& \geq C t^{-\left(\frac{3}{2}+s\right)} \sum_{k=\left[\frac{\eta \sqrt{t}}{\pi}\right]+1}^{\left[\frac{2 \eta \sqrt{t}}{\pi}\right]+1} \int_{\frac{k \pi+\frac{\pi}{6}}{\sqrt{t}}}^{\frac{k \pi+\frac{\pi}{3}}{\sqrt{t}}} e^{-(\mu+\nu) R^{2}} R^{2 s+2} \mathrm{~d} R \\
& \geq C t^{-\left(\frac{3}{2}+s\right)},
\end{aligned}
$$

where there exits a positive large time $t_{*}$ such that for $t \geq t_{*}$, we can obtain

$$
e^{-(\mu+\nu) R^{2}} \geq e^{-(\mu+\nu)\left(\frac{k \pi+\frac{\pi}{3}}{\sqrt{t}}\right)^{2}} \geq e^{-(\mu+\nu)\left(\frac{\left.\left(\frac{2 \eta \sqrt{t}}{\pi}\right]+1\right) \pi+\frac{\pi}{3}}{\sqrt{t}}\right)^{2}} \geq C,
$$

with $C$ a positive constant that independent of time.

Proof of inequality (2.21). Multiplying the first and second equations of (2.19) by $\varrho_{\delta}$ and $m_{\delta}$, respectively, it holds on

$$
\frac{1}{2} \frac{\mathrm{d}}{\mathrm{d} t} \int\left(\left|\varrho_{\delta}\right|^{2}+\left|m_{\delta}\right|^{2}\right) \mathrm{d} x+\mu \int\left|\nabla m_{\delta}\right|^{2} \mathrm{~d} x+(\mu+\nu) \int\left|\operatorname{div} m_{\delta}\right|^{2} \mathrm{~d} x-\kappa \int \nabla \Delta \varrho_{\delta} \cdot m_{\delta} \mathrm{d} x=\int S \cdot \nabla m_{\delta} \mathrm{d} x .
$$

Due to the fact that $P^{\prime}(1)=1$, we can use the Taylor expression formula to get

$$
P(1+\varrho)-P(1)-\varrho \sim \varrho^{2},
$$

which, together with Sobolev's inequality, yields directly

$$
\|S\|_{L^{2}} \leq C\|(\varrho, u)\|_{H^{2}}\left(\|\nabla \varrho\|_{H^{1}}+\|\nabla u\|_{L^{2}}\right)
$$

where the symbol $\sim$ represents the equivalent relation. By virtue of integrating by parts and using the transport equation, it is easy to get

$$
\int \nabla \Delta \varrho_{\delta} \cdot m_{\delta} \mathrm{d} x=-\frac{1}{2} \frac{\mathrm{d}}{\mathrm{d} t} \int\left|\nabla \varrho_{\delta}\right|^{2} \mathrm{~d} x
$$

Then, we get

$$
\frac{\mathrm{d}}{\mathrm{d} t}\left(\left\|\left(\varrho_{\delta}, m_{\delta}\right)\right\|_{L^{2}}^{2}+\kappa\left\|\nabla \varrho_{\delta}\right\|_{L^{2}}^{2}\right)+\mu\left\|\nabla m_{\delta}\right\|_{L^{2}}^{2}+\nu\left\|\operatorname{div} m_{\delta}\right\|_{L^{2}}^{2} \mathrm{~d} x \leq C\|(\varrho, u)\|_{H^{2}}^{2}\left(\|\nabla \varrho\|_{H^{1}}^{2}+\|u\|_{L^{2}}^{2}\right) .
$$


Applying the equation (2.19), it is easy to obtain for $k=1,2,3$,

$$
\begin{aligned}
& \frac{\mathrm{d}}{\mathrm{d} t}\left\|\nabla^{k}\left(\varrho_{\delta}, m_{\delta}\right)\right\|_{L^{2}}^{2}+\mu\left\|\nabla^{k+1} m_{\delta}\right\|_{L^{2}}^{2}+(\mu+\nu)\left\|\nabla^{k} \operatorname{div} m_{\delta}\right\|_{L^{2}}^{2}-\kappa \int \nabla^{k+1} \Delta \varrho_{\delta} \cdot \nabla^{k} m_{\delta} \mathrm{d} x \\
& \quad \leq\left\|\nabla^{k} S\right\|_{L^{2}}\left\|\nabla^{k+1} m_{\delta}\right\|_{L^{2}} .
\end{aligned}
$$

At first, we estimate the term $\int \nabla^{k+1} \Delta \varrho_{\delta} \cdot \nabla^{k} m_{\delta} \mathrm{d} x$ for $k=1,2,3$. Using integration by parts and transport equation, we can obtain

$$
\int \nabla^{k+1} \Delta \varrho_{\delta} \cdot \nabla^{k} m_{\delta} \mathrm{d} x=-\frac{1}{2} \frac{\mathrm{d}}{\mathrm{d} t} \int\left|\nabla^{k+1} \varrho_{\delta}\right|^{2} \mathrm{~d} x .
$$

Now we give the estimates for $\left\|\nabla^{k} S\right\|_{L^{2}}^{2}, k=1,2,3$. Indeed, when $k=1$, we apply Sobolev's inequality to obtain

$$
\begin{aligned}
\|\nabla((1+\varrho) u \otimes u)\|_{L^{2}} & \leq C\|1+\varrho\|_{L^{\infty}}\|u\|_{L^{\infty}}\|\nabla u\|_{L^{2}}+C\|u\|_{L^{\infty}}^{2}\|\nabla \varrho\|_{L^{2}} \\
& \leq C\left(1+\|\nabla u\|_{H^{1}}\right)\|\nabla u\|_{H^{1}}\|\nabla(\varrho, u)\|_{L^{2}} \\
& \leq C\|\nabla u\|_{H^{1}}\|\nabla(\varrho, u)\|_{L^{2}} .
\end{aligned}
$$

Similarly, we also have for $k=1$,

$$
\left\|\nabla^{2}(\varrho u)\right\|_{L^{2}}+\|\nabla \operatorname{div}(\varrho u)\|_{L^{2}} \leq C\|\nabla(\varrho, u)\|_{H^{1}}\left\|\nabla^{2}(\varrho, u)\right\|_{L^{2}} .
$$

By the same way, we have

$$
\left\|\nabla\left(\varrho \nabla^{2} \varrho\right)\right\|_{L^{2}} \leq C\left\|\nabla^{2} \varrho\right\|_{L^{2}}\left\|\nabla^{2} \varrho\right\|_{H^{1}}+C\|\nabla \varrho\|_{H^{1}}\left\|\nabla^{3} \varrho\right\|_{L^{2}}
$$

and

$$
\left\|\nabla|\nabla \varrho|^{2}\right\|_{L^{2}} \leq C\left\|\nabla^{2} \varrho\right\|_{L^{2}}\left\|\nabla^{2} \varrho\right\|_{H^{1}}
$$

By virtue of the Taylor expression formula, we get

$$
\nabla(P(1+\varrho)-P(1)-\varrho)=P^{\prime}(1+\varrho) \nabla \varrho-P^{\prime}(1) \nabla \varrho \sim \varrho \nabla \varrho,
$$

where we have used the fact that $P^{\prime}(1)=1$. Then, we use Sobolev's inequality to obtain

$$
\|\nabla(P(1+\varrho)-P(1)-\varrho)\|_{L^{2}} \leq C\|\nabla \varrho\|_{H^{1}}\|\nabla \varrho\|_{L^{2}} .
$$

Thus, it holds on for $k=1$,

$$
\|\nabla S\|_{L^{2}} \leq C\|\nabla(\varrho, u)\|_{H^{1}}\left(\|\nabla \varrho\|_{H^{2}}+\|\nabla u\|_{H^{1}}\right) .
$$

Then, we use Cauchy inequality to get

$$
\frac{\mathrm{d}}{\mathrm{d} t}\left(\left\|\nabla\left(m_{\delta}, \varrho_{\delta}\right)\right\|_{L^{2}}^{2}+\kappa\left\|\nabla^{2} \varrho_{\delta}\right\|_{L^{2}}^{2}\right)+\mu\left\|\nabla^{2} m_{\delta}\right\|_{L^{2}}^{2} \leq C\|\nabla(\varrho, u)\|_{H^{1}}^{2}\left(\left\|\nabla^{2} \varrho\right\|_{H^{1}}^{2}+\|\nabla u\|_{H^{1}}^{2}\right) .
$$

Notice that

$$
\begin{aligned}
\nabla^{2}(P(1+\varrho)-P(1)-\varrho) & =P^{\prime \prime}(1+\varrho) \nabla \varrho \nabla \varrho+P^{\prime}(1+\varrho) \nabla^{2} \varrho-P^{\prime}(1) \nabla^{2} \varrho \\
& \sim \nabla \varrho \nabla \varrho+\varrho \nabla^{2} \varrho,
\end{aligned}
$$

and

$$
\begin{aligned}
& \nabla^{3}(P(1+\varrho)-P(1)-\varrho) \\
& \quad=2 P^{\prime \prime \prime}(1+\varrho) \nabla \varrho \nabla \varrho \nabla \varrho+2 P^{\prime \prime}(1+\varrho) \nabla^{2} \varrho \nabla \varrho+P^{\prime}(1+\varrho) \nabla^{3} \varrho-P^{\prime}(1) \nabla^{3} \varrho \\
& \quad \sim \nabla \varrho \nabla \varrho \nabla \varrho+\nabla \varrho \nabla^{2} \varrho+\varrho \nabla^{3} \varrho .
\end{aligned}
$$

Next, employing Sobolev's inequality, it is easy to get

$$
\left\|\nabla^{2} S\right\|_{L^{2}} \leq C\left\|\nabla^{2}(\varrho, u)\right\|_{H^{1}}\left(\|\nabla \varrho\|_{H^{2}}+\|\nabla u\|_{H^{1}}\right)+C\|\nabla \varrho\|_{H^{1}}\left\|\nabla^{4} \varrho\right\|_{L^{2}},
$$

and

$$
\left\|\nabla^{3} S\right\|_{L^{2}} \leq C\left\|\nabla^{3}(\varrho, u)\right\|_{L^{2}}\left(\|\nabla \varrho\|_{H^{2}}+\|\nabla u\|_{H^{1}}\right)+C\|\nabla \varrho\|_{H^{1}}\left(\left\|\nabla^{5} \varrho\right\|_{L^{2}}+\left\|\nabla^{4} u\right\|_{L^{2}}\right) .
$$


Then, we obtain the following estimates

$$
\begin{aligned}
& \frac{\mathrm{d}}{\mathrm{d} t}\left(\left\|\nabla^{2}\left(m_{\delta}, \varrho \delta\right)\right\|_{L^{2}}^{2}+\kappa\left\|\nabla^{3} \varrho_{\delta}\right\|_{L^{2}}^{2}\right)+\mu\left\|\nabla^{3} m_{\delta}\right\|_{L^{2}}^{2} \\
& \quad \leq C\left\|\nabla^{2}(\varrho, u)\right\|_{H^{1}}^{2}\left(\|\nabla \varrho\|_{H^{2}}^{2}+\|\nabla u\|_{H^{1}}^{2}\right)+C\|\nabla \varrho\|_{H^{1}}^{2}\left\|\nabla^{4} \varrho\right\|_{L^{2}}^{2},
\end{aligned}
$$

and

$$
\begin{aligned}
& \frac{\mathrm{d}}{\mathrm{d} t}\left(\left\|\nabla^{3}\left(m_{\delta}, \varrho \delta\right)\right\|_{L^{2}}^{2}+\kappa\left\|\nabla^{4} \varrho_{\delta}\right\|_{L^{2}}^{2}\right)+\mu\left\|\nabla^{4} m_{\delta}\right\|_{L^{2}}^{2} \\
& \quad \leq C\left\|\nabla^{3}(\varrho, u)\right\|_{L^{2}}^{2}\left(\|\nabla \varrho\|_{H^{2}}^{2}+\|\nabla u\|_{H^{1}}^{2}\right)+C\|\nabla \varrho\|_{H^{1}}^{2}\left(\left\|\nabla^{5} \varrho\right\|_{L^{2}}^{2}+\left\|\nabla^{4} u\right\|_{L^{2}}^{2}\right) .
\end{aligned}
$$

Therefore, we complete the proof of claim estimate (2.21).

Proof of inequality (2.22). Taking $k(k=0,1,2,3)$ th spatial derivatives to the second equation of (2.19) and multiplying the equation by $\nabla^{k+1} \varrho_{\delta}$, then we have

$$
\begin{aligned}
& \int \partial_{t} \nabla^{k} m_{\delta} \cdot \nabla^{k+1} \varrho_{\delta} \mathrm{d} x+\int\left|\nabla^{k+1} \varrho_{\delta}\right|^{2} \mathrm{~d} x-\kappa \int \nabla^{k+1} \Delta \varrho_{\delta} \cdot \nabla^{k+1} \varrho_{\delta} \mathrm{d} x \\
& =\int\left(\mu \nabla^{k} \Delta m_{\delta}+(\mu+\nu) \nabla^{k+1} \operatorname{div} m_{\delta}\right) \cdot \nabla^{k+1} \varrho_{\delta} \mathrm{d} x-\int \nabla^{k} \operatorname{div} S \cdot \nabla^{k+1} \varrho_{\delta} \mathrm{d} x .
\end{aligned}
$$

Using the first equation of (2.19), it holds on

$$
\begin{aligned}
\int \partial_{t} \nabla^{k} m_{\delta} \cdot \nabla^{k+1} \varrho_{\delta} \mathrm{d} x & =\frac{\mathrm{d}}{\mathrm{d} t} \int \nabla^{k} m_{\delta} \cdot \nabla^{k+1} \varrho_{\delta} \mathrm{d} x-\int \nabla^{k} m_{\delta} \cdot \nabla^{k+1} \partial_{t} \varrho_{\delta} \mathrm{d} x \\
& =\frac{\mathrm{d}}{\mathrm{d} t} \int \nabla^{k} m_{\delta} \cdot \nabla^{k+1} \varrho_{\delta} \mathrm{d} x+\int \nabla^{k} m_{\delta} \cdot \nabla^{k+1} \operatorname{div} m_{\delta} \mathrm{d} x \\
& =\frac{\mathrm{d}}{\mathrm{d} t} \int \nabla^{k} m_{\delta} \cdot \nabla^{k+1} \varrho_{\delta} \mathrm{d} x-\int\left|\nabla^{k} \operatorname{div} m_{\delta}\right|^{2} \mathrm{~d} x
\end{aligned}
$$

After integration by part, it holds on

$$
\int \nabla^{k+1} \Delta \varrho_{\delta} \cdot \nabla^{k+1} \varrho_{\delta} \mathrm{d} x=-\int\left|\nabla^{k+2} \varrho_{\delta}\right|^{2} \mathrm{~d} x .
$$

Thus, we combine the above three equalities to obtain

$$
\begin{aligned}
& \frac{\mathrm{d}}{\mathrm{d} t} \int \nabla^{k} m_{\delta} \cdot \nabla^{k+1} \varrho_{\delta} \mathrm{d} x+\int\left|\nabla^{k+1} \varrho_{\delta}\right|^{2} \mathrm{~d} x+\kappa \int\left|\nabla^{k+2} \varrho_{\delta}\right|^{2} \mathrm{~d} x \\
& \quad=\int\left|\nabla^{k} \operatorname{div} m_{\delta}\right|^{2} \mathrm{~d} x-\int \nabla^{k} \operatorname{div} S \cdot \nabla^{k+1} \varrho_{\delta} \mathrm{d} x+\int\left(\mu \nabla^{k} \Delta m_{\delta}+(\mu+\nu) \nabla^{k+1} \operatorname{div} m_{\delta}\right) \cdot \nabla^{k+1} \varrho_{\delta} \mathrm{d} x,
\end{aligned}
$$

which, together with integration by parts and Cauchy inequality, yields directly

$$
\frac{\mathrm{d}}{\mathrm{d} t} \int \nabla^{k} m_{\delta} \cdot \nabla^{k+1} \varrho_{\delta} \mathrm{d} x+\int\left|\nabla^{k+1} \varrho_{\delta}\right|^{2} \mathrm{~d} x+\frac{1}{2} \kappa \int\left|\nabla^{k+2} \varrho_{\delta}\right|^{2} \mathrm{~d} x \leq C\left(\left\|\nabla^{k+1} m_{\delta}\right\|_{L^{2}}^{2}+\left\|\nabla^{k} S\right\|_{L^{2}}^{2}\right),
$$

which implies (2.22). Therefore, we complete proof of claim estimate (2.22).

Proof of inequality (2.30). It is easy to see that

$$
\int_{0}^{t} e^{-\frac{C}{C^{*}}(t-\tau)}(1+\tau)^{-\frac{5}{2}}\left(\left\|\nabla^{4} \varrho\right\|_{H^{1}}^{2}+\left\|\nabla^{4} u\right\|_{L^{2}}^{2}\right) \mathrm{d} \tau \leq \int_{0}^{t} e^{-\frac{C}{C^{*}}(t-\tau)}\left(\left\|\nabla^{4} \varrho\right\|_{H^{1}}^{2}+\left\|\nabla^{4} u\right\|_{L^{2}}^{2}\right) \mathrm{d} \tau .
$$

In [10], the following estimate holds on

$$
\frac{\mathrm{d}}{\mathrm{d} t} \mathcal{F}_{l}^{3}(t)+C\left(\left\|\nabla^{l+1} \varrho\right\|_{H^{4-l}}^{2}+\left\|\nabla^{l+1} u\right\|_{H^{3-l}}^{2}\right) \leq 0, \quad l=0,1,2,3,
$$


where

$$
\mathcal{F}_{l}^{3}(t):=\left\|\nabla^{l} \varrho\right\|_{H^{4-l}}^{2}+\left\|\nabla^{l} u\right\|_{H^{3-l}}^{2}+\delta \sum_{k=l}^{3} \int \nabla^{k} u \cdot \nabla^{k+1} \varrho \mathrm{d} x,
$$

and $\delta$ is a small positive number. By multiplying the inequality (3.1) with $l=3$ by $e^{\frac{C}{C^{*}} t}$, it holds on

$$
\begin{aligned}
& \frac{\mathrm{d}}{\mathrm{d} t}\left(e^{\frac{C}{C^{*}} t} \mathcal{F}_{3}^{3}(t)\right)+C e^{\frac{C}{C^{*}} t}\left(\left\|\nabla^{4} \varrho\right\|_{H^{1}}^{2}+\left\|\nabla^{4} u\right\|_{L^{2}}^{2}\right) \\
& \quad \leq C e^{\frac{C}{C^{*}} t}\left(\left\|\nabla^{3} \varrho\right\|_{H^{1}}^{2}+\left\|\nabla^{3} u\right\|_{L^{2}}^{2}\right) ;
\end{aligned}
$$

then, by integrating about time over $[0, t]$, similar to $(2.29)$, one arrives at

$$
\begin{aligned}
& \mathcal{F}_{3}^{3}(t)+\int_{0}^{t} e^{-\frac{C}{C^{*}}(t-\tau)}\left(\left\|\nabla^{4} \varrho(\tau)\right\|_{H^{1}}^{2}+\left\|\nabla^{4} u(\tau)\right\|_{L^{2}}^{2}\right) \mathrm{d} \tau \\
& \leq C e^{-\frac{C}{C^{*}} t} \mathcal{F}_{3}^{3}(0)+C \int_{0}^{t} e^{-\frac{C}{C^{*}}(t-\tau)}\left(\left\|\nabla^{3} \varrho(\tau)\right\|_{H^{1}}^{2}+\left\|\nabla^{3} u(\tau)\right\|_{L^{2}}^{2}\right) \mathrm{d} \tau \\
& \leq C e^{-\frac{C}{C^{*}} t}\left(\left\|\nabla^{3} \varrho_{0}\right\|_{H^{1}}^{2}+\left\|\nabla^{3} u_{0}\right\|_{L^{2}}^{2}\right)+C \int_{0}^{t} e^{-\frac{C}{C^{*}}(t-\tau)}\left(\left\|\nabla^{3} \varrho(\tau)\right\|_{H^{1}}^{2}+\left\|\nabla^{3} u(\tau)\right\|_{L^{2}}^{2}\right) \mathrm{d} \tau \\
& \quad \leq C e^{-\frac{C}{C^{*}} t}+C \int_{0}^{t} e^{-\frac{C}{C^{*}}(t-\tau)}(1+\tau)^{-\frac{9}{2}} \mathrm{~d} \tau \\
& \leq C e^{-\frac{C}{C^{*}} t}+(1+t)^{-\frac{9}{2}} \\
& \leq C(1+t)^{-\frac{9}{2}}
\end{aligned}
$$

where we utilize decay estimate (1.7). Therefore, we complete proof of claim estimate (2.30).

Proof of inequality (2.36). Replacing $l$ by $l+1$ in (3.1), and then multiplying both sides by $(1+t)^{l+\frac{3}{2}}$, one arrives at

$$
\begin{aligned}
& \frac{\mathrm{d}}{\mathrm{d} t}\left[(1+t)^{l+\frac{3}{2}} \mathcal{F}_{l+1}^{3}(t)\right]+C(1+t)^{l+\frac{3}{2}}\left(\left\|\nabla^{l+2} \varrho\right\|_{H^{3-l}}^{2}+\left\|\nabla^{l+2} u\right\|_{H^{2-l}}^{2}\right) \\
& \quad \leq C(1+t)^{l+\frac{1}{2}} \mathcal{F}_{l+1}^{3}(t) .
\end{aligned}
$$

The integration of the above inequality with respect to time over $[0, t]$ implies that

$$
\begin{aligned}
& \int_{0}^{t}(1+\tau)^{l+\frac{3}{2}}\left(\left\|\nabla^{l+2} \varrho(\tau)\right\|_{H^{3-l}}^{2}+\left\|\nabla^{l+2} u(\tau)\right\|_{H^{2-l}}^{2}\right) \mathrm{d} \tau \\
& \quad \leq C \mathcal{F}_{l+1}^{3}(0)+C \int_{0}^{t}(1+\tau)^{l+\frac{1}{2}} \mathcal{E}_{l+1}^{3}(\tau) \mathrm{d} \tau \\
& \quad \leq C+C \int_{0}^{t}(1+\tau)^{l+\frac{1}{2}}(1+\tau)^{-\frac{5+2 l}{2}} \mathrm{~d} \tau \\
& \leq C,
\end{aligned}
$$

where we used the estimate $\mathcal{F}_{l}^{3}(t) \leq C(1+t)^{-\frac{3+2 l}{2}}$ for $l=0,1,2,3$. Therefore, we complete proof of claim estimate (2.36). 


\section{Acknowledgements}

This research was partially supported by NSFC (Grant Nos. 11801586, 11971496 and 11431015) and the Fundamental Research Funds for the Central Universities of China (Grant No. 18lgpy66).

Open Access. This article is licensed under a Creative Commons Attribution 4.0 International License, which permits use, sharing, adaptation, distribution and reproduction in any medium or format, as long as you give appropriate credit to the original author(s) and the source, provide a link to the Creative Commons licence, and indicate if changes were made. The images or other third party material in this article are included in the article's Creative Commons licence, unless indicated otherwise in a credit line to the material. If material is not included in the article's Creative Commons licence and your intended use is not permitted by statutory regulation or exceeds the permitted use, you will need to obtain permission directly from the copyright holder. To view a copy of this licence, visit http://creativecommons.org/licenses/by/4.0/.

Publisher's Note Springer Nature remains neutral with regard to jurisdictional claims in published maps and institutional affiliations.

\section{References}

[1] Bian, D.F., Yao, L., Zhu, C.J.: Vanishing capillarity limit of the compressible fluid models of Korteweg type to the Navier-Stokes equations. SIAM J. Math. Anal. 46(2), 1633-1650 (2014)

[2] Bresch, D., Desjardins, B., Lin, C.K.: On some compressile fluid models: Korteweg, lubrication and hallow water systems. Commun. Partial Differ. Equ. 28(3-4), 843-868 (2003)

[3] Charve, F., Haspot, B.: Existence of a global strong solution and vanishing capillarity-viscosity limit in one dimension for the Korteweg system. SIAM J. Math. Anal. 45(2), 469-494 (2013)

[4] Chen, Z.Z., Zhao, H.J.: Existence and nonlinear stability of stationary solutions to the full compressible Navier-StokesKorteweg system. J. Math. Pures Appl. (9) 101(3), 330-371 (2014)

[5] Chen, Z.Z., Chai, X.J., Dong, B.Q., Zhao, H.J.: Global classical solutions to the one-dimensional compressible fluid models of Korteweg type with large initial data. J. Differ. Equ. 259, 4376-4411 (2015)

[6] Chikami, N., Kobayashi, T.: Global well-posedness and time-decay estimates of the compressible Navier-StokesKorteweg system in critical Besov spaces. J. Math. Fluid Mech. 21(2), 32 (2019)

[7] Danchin, R., Desjardins, B.: Existence of solutions for compressible fluid models of Korteweg type. Ann. Inst. Henri Poincaré Anal. Non Linéaire 18(1), 97-133 (2001)

[8] Dunn, J., Serrin, J.: On the thermomechanics of interstitial working. Arch. Ration. Mech. Anal. 88(2), 95-133 (1985)

[9] Gao, J.C., Lyu, Z.Y., Yao, Z.A.: Lower bound and space-time decay rates of higher order derivatives of solution for the compressible Navier-Stokes and Hall-MHD equations. arXiv:1909.13269

[10] Gao, J.C., Zou, Y., Yao, Z.A.: Long-time behavior of solution for the compressible Navier-Stokes-Korteweg equations in $\mathbb{R}^{3}$. Appl. Math. Lett. 48, 30-35 (2015)

[11] Haspot, B.: Existence of global weak solution for compressible fluid models of Korteweg type. J. Math. Fluid Mech. 13(2), 223-249 (2011)

[12] Hattori, H., Li, D.N.: Solutions for two-dimensional system for materials of Korteweg type. SIAM J. Math. Anal. 25(1), 85-98 (1994)

[13] Hattori, H., Li, D.N.: Global solutions of a high-dimensional system for Korteweg materials. J. Math. Anal. Appl. 198(1), 84-97 (1996)

[14] Hattori, H., Li, D.N.: The existence of global solutions to a fluid dynamic model for materials for Korteweg type. J. Partial Differ. Equ. 9(4), 323-342 (1996)

[15] Hou, X.F., Peng, H.Y., Zhu, C.J.: Global classical solutions to the 3D Navier-Stokes-Korteweg equations with small initial energy. Anal. Appl. (Singap.) 16(1), 55-84 (2018)

[16] Kotschote, M.: Strong solutions for a compressible fluid model of Korteweg type. Ann. Inst. Henri Poincare Non Linear Anal. 25(4), 679-696 (2008)

[17] Korteweg, D.J.: Sur la forme que prennent les équations du mouvement des fluids si I'on tient compte des forces capillaires causés par les variation de densité. Arch. Néer. Sci. Exactes Sér. II(6), 1-24 (1901)

[18] Li, Y.P.: Global existence and optimal decay rate of the compressible Navier-Stokes-Korteweg equations with external force. J. Math. Anal. Appl. 388(2), 1218-1232 (2012)

[19] Schonbek, M.E.: $L^{2}$ decay for weak solutions of the Navier-Stokes equations. Arch. Ration. Mech. Anal. 88, 209-222 (1985) 
[20] Tan, Z., Wang, H.Q., Xu, J.K.: Global existence and optimal $L^{2}$ decay rate for the strong solutions to the compressible fluid models of Korteweg type. J. Math. Anal. Appl. 390(1), 181-187 (2012)

[21] Tan, Z., Wang, Y.J.: Strong solutions for the incompressible fluid models of Korteweg type. Acta Math. Sci. Ser. B Engl. Ed. 30, 799-809 (2010)

[22] Tan, Z., Zhang, R.F.: Optimal decay rates of the compressible fluid models of Korteweg type. Z. Angew. Math. Phys. 65, 279-300 (2014)

[23] Waals, J.D.: Thermodynamische Theorie der Kapillarität unter Voraussetzung stetiger Dichteänderung. Z. Phys. Chem. 13, 657-725 (1894)

[24] Wang, W.J., Wang, W.K.: Decay rates of the compressible Navier-Stokes-Korteweg equations with potential forces. Discrete Contin. Dyn. Syst. 35, 513-536 (2015)

[25] Wang, Y.J., Tan, Z.: Optimal decay rates for the compressible fluid models of Korteweg type. J. Math. Anal. Appl. 379(1), 256-271 (2011)

Jincheng Gao, Zeyu Lyu and Zheng-an Yao

School of Mathematics

Sun Yat-Sen University

Guangzhou 510275

People's Republic of China

e-mail: lvzy3@mail2.sysu.edu.cn

Jincheng Gao

e-mail: gaojc1998@163.com

Zheng-an Yao

e-mail: mcsyao@mail.sysu.edu.cn

(Received: December 26, 2019) 